\title{
Radiation fields in the Schwarzschild background*
}

\author{
James M. Bardeen $\dagger$ \\ University of Washington, Seattle, Washington
}

\begin{abstract}
William H. Press $\ddagger$
California Institute of Technology, Pasadena, California

(Received 29 March 1972; revised manuscript received 16 August 1972)

Scalar, electromagnetic, and gravitational test fields in the Schwarzschild background are examined with the help of the general retarded solution of a single master wave equation. The solution for each multipole is generated by a single arbitrary function of retarded time, the retarded multipole moment. We impose only those restrictions on the time dependence of the multipole moment which are required for physical regularity. We find physically well-behaved solutions which (i) do not satisfy the Penrose peeling theorems at past null infinity and/or (ii) do not have well-defined Newman-Penrose quantities. Even when the NP quantities exist, they are not measurable; they represent an "average" multipole moment over the infinite past, and their conservation is essentially trivial.
\end{abstract}

\section{INTRODUCTION}

Two general relativistic effects make it difficult to study the exact propagation of radiation fields. First, the curvature of the space-time manifold influences the propagation of the radiation. Second, the stressenergy of the radiation acts to produce curvature in the manifold. Acting in concert, these effects produce a nonlinear theory, with an extreme dearth of known, exact radiation solutions available for study.

In studying gravitational waves, it has frequently been useful to use the "linearized theory," in which the manifold is taken to be flat, and the waves are sufficiently weak that they do not destroy the flatness. Unfortunately, certain interesting phenomena vanish in the linearized case. For example, in general the propagation of radiation is not entirely along null characteristics, as Kundt and Newman ${ }^{1}$ have shown for scalar and electromagnetic test fields in the Schwarzschild metric, as McLenaghan ${ }^{2}$ has shown for scalar test fields in any nonflat background satisfying the vacuum Einstein equations, and as Bonnor and Rotenberg ${ }^{3}$ have shown for asymptotically flat gravitational fields. The radiation backscatters off of nonuniformities in the curvature of the background space-time. For example, there is generally backscatter left behind a burst of outgoing radiation. Although the backscatter dies off in time at fixed radius, the field at any point in space does not become exactly static in a finite retarded time. Certain coefficients associated with the asymptotic field near future null infinity, the Newman-Penrose quantities (NPQ's) 4,5 are related to the backscatter from outgoing waves. In a flat background these coefficients measure properties of incoming waves and vanish identically when an outgoing-wave boundary condition is imposed. In curved space, the Einstein-Maxwell equations appear to guarantee that the NPQ's are conserved for dynamic fields; but investigations of their physical significance have been hampered by the absence of exact solutions with nontrivial NPQ's.

Backscatter and nontrivial NPQ's do not require the full nonlinear theory. They require that the background influence the radiation, but not vice versa. Thus they can be studied in detail for fields which are linearized about (i.e., weakly superimposed on) a curved background. The work of Price 6.7 on the behavior of integer-spin test fields in the collapse of a slightly nonspherical star, has furnished the key to this sort of an approach.

We have used Price's equations to analyze in detail the propagation of scalar, electromagnetic, and gravitational test fields in the Schwarzschild background. The sources of the fields are assumed to remain bounded for all time inside a radius $R>2 M$, where $M$ is the gravitational mass (units with $c=G=1$ ). We exhibit a single partial differential equation which fully describes the radiative part of the various test fields, and we solve this master equation for the general retarded solution in the region $r>R$. The solution is an expansion in powers of $(2 M / r)$ which converges uniformly in this region at all retarded times.

With the general solution, we are able to examine the backscatter in some detail and to elucidate the nature and physical significance of the NPQ's. The solution also sheds considerable light on the "peeling theorems," which deal with the asymptotic radiation field at null infinity.

Our results for the NPQ's have been reported previous$1 y^{8}$; in this paper they are amplified from a somewhat different viewpoint. We find that the NPQ's do not always exist (i.e., the limits defining them do not always converge). When they do exist, they are a certain average of the value of the source's lowest radiatable multipole moment over the infinite past. The presence of this "average value" in the field is due to the superposition of backscatter from the outgoing radiation of all previous epochs. The conservation of the NPQ's has a simple interpretation: The contribution of the present finite epoch to the average of the infinite past is vanishingly small.

An important point is that the NPQ's, even when they exist, are not operationally measurable. An observational measurement of finite accuracy and duration, and at finite radius, can at best determine a quantity (we here call it a measurable NPQ or MNPQ) which is an average over the recent past (this is made precise in Sec.6); and there is no observational way to tell whether this average agrees with the "primordial" NPQ or not.

Most previous theorems dealing with the asymptotic behavior of the fields at null infinity and, in particular, the peeling theorem of Penrose ${ }^{9}$ based on a conformal treatment of infinity, ${ }^{10}$ make certain mathematical regularity assumptions. For instance, Penrose assumes that the conformally transformed space-time manifold is $C^{4}$-differentiable everywhere (including future and past null infinity), with a $C^{3}$ metric. We ask, for retarded test fields in the Schwarzschild background whether all physically acceptable solutions of the field 
equations are consistent with the assumptions of the Penrose theorem. The answer is no. Only solutions for which the gravitational quadrupole moment is asymptotically static in the infinite past satisfy the theorem for the gravitational field at past null infinity. Our general retarded field is consistent with the peeling theorem at future null infinity. We show that all solutions which are asymptotically regular in the Penrose sense at past null infinity possess $N P Q$ 's at future null infinity.

The mathematical foundation of this paper is the Newman-Penrose spin coefficient formalism, as adapted by Price ${ }^{7}$ for test fields in the Schwarzschild metric. This is reviewed briefly in Sec. 2 , and the equations for scalar, electromagnetic, and gravitational test fields are given. Section 3 solves these equations as special cases of a single "master" equation. Section 4 deals with the solutions for the lowest radiatable moment; Sec. 5 with the peeling theorems; and Sec. 6 the NewmanPenrose quantities.

\section{FORMAL PRELIMINARIES}

The conventional form of the Schwarzschild metric is

$$
\begin{aligned}
d s^{2}=(1-2 M / r) d t^{2}-(1- & 2 M / r)^{-1} d r^{2} \\
& -r^{2}\left(d \theta+\sin ^{2} \theta d \varphi^{2}\right) .
\end{aligned}
$$

Outgoing null geodesics are the surfaces of constant $u$, $\theta, \varphi$, where $u$ is the retarded time

$$
u \equiv t-r-2 M \ln (r / 2 M-1) \equiv t-r^{*},
$$

while for ingoing radial null geodesics,

$$
v \equiv t+r+2 M \ln (r / 2 M-1) \equiv t+r^{*}=\text { const. }
$$

The radius $r$ is both the proper circumferential radius governing the area of 2-spheres and an affine parameter along the radial null geodesics. We will always impose a boundary condition that there be no "free" incoming waves, so it is convenient to use the retarded time $u$ and the radius $r$ as coordinates. Then the metric is

$d s^{2}=(1-2 M / r) d u^{2}+2 d u d r-r^{2}\left(d \theta^{2}+\sin ^{2} \theta d \varphi^{2}\right)$.

The Newman-Penrose spin coefficient formalism 11 is a powerful method for dealing with radiation in asymptotically flat space-times. It is based on a tetrad of complex, null 4-vectors $l^{\mu}, n^{\mu}, m^{\mu}, m^{*}$ satisfying

$$
l \cdot n=-m \cdot m^{*}=1
$$

with all other dot products zero. All tensors can be reduced to (in general) complex scalars by contraction with members of this null tetrad. The "spin coefficients" are scalars constructed from covariant derivatives of the tetrad vectors. Newman-Penrose scalars have a conformal weight $c$ and a spin weight $p$ if under the transformation

$$
\tilde{l}^{\mu}=\lambda l^{\mu}, \quad \tilde{n^{\mu}}=\lambda^{-1} n^{\mu}, \quad \tilde{m}^{\mu}=e^{i \pi} m^{\mu},
$$

the scalar $T$ transforms as

$$
\tau=\lambda^{c} e^{i p \eta T} \text {. }
$$

In the Schwarzschild background a special choice for the null tetrad which simplifies the spin coefficients is, in $u, r, \theta, \varphi$ coordinates,

$$
\begin{aligned}
& l^{\mu}=[0,1,0,0], \\
& n^{\mu}=\left[1,-\frac{1}{2}(1-2 M / r), 0,0\right], \\
& m^{\mu}=(1 / \sqrt{ } 2)[0,0,1 / r, i /(r \sin \theta)] .
\end{aligned}
$$

Thus, $l^{\mu}$ is tangent to outgoing radial null geodesics and $n^{\mu}$ is tangent to ingoing radial null geodesics.

The physically measurable tensor associated with the electromagnetic field is the electromagnetic field tensor $F_{\mu \nu}$; that associated with the free gravitational field is the Weyl tensor $C_{\alpha \beta \gamma \delta}$ (in vacuum, identical to the Riemann tensor $\left.R_{\alpha \beta \gamma \delta}\right)$. The tetrad (2.8) is contracted with $F_{\mu \nu}$ to obtain the NP scalars for a test electromagnetic field

$$
\begin{aligned}
& \Phi_{1}=F_{\mu \nu} l^{\mu} m^{\nu}, \\
& \Phi_{0}=\frac{1}{2} F_{\mu \nu}\left(l^{\mu} n^{\nu}-m^{\mu} m^{* \nu}\right), \\
& \Phi_{-1}=F_{\mu \nu} m^{* \mu} n^{\nu} .
\end{aligned}
$$

Fortuitously, the subscript denotes both the spin weight and the conformal weight of the scalar. In terms of the physical electric and magnetic field components measured by an observer at rest in the Schwarzschild metric (2.1),

$$
\begin{aligned}
\Phi_{+1}=2^{-1 / 2}(1-2 M / r)^{-1 / 2}\left[E^{(\theta)}-B^{(\varphi)}+i\left(E^{(\varphi)}+B^{(\theta)}\right)\right] \\
\Phi_{0}=-\frac{1}{2}\left(E^{(r)}+i B^{(r)}\right) \\
\Phi_{-1}=-2^{-3 / 2}(1-2 M / r)^{1 / 2}\left[E^{(\theta)}+B^{(\varphi)}-i\left(E^{(\varphi)}-B^{(\theta)}\right)\right] .
\end{aligned}
$$

The $\Phi_{p}$ are completely equivalent to $F_{\mu \nu}$-each contains six independent real functions.

Similarly, there are five NP scalars containing ten independent real functions which are equivalent in information content to the Weyl tensor:

$$
\begin{aligned}
\Psi_{2} & =-C_{\alpha \beta \gamma \delta} l^{\alpha_{m}^{\beta} l \gamma m^{\delta},} \quad \Psi_{1}=-C_{\alpha \beta \gamma \delta} l^{\alpha} n^{\beta} l \gamma m^{\delta}, \\
\Psi_{0} & =-C_{\alpha \beta \gamma \delta} l^{\alpha} m^{\beta} m^{* \gamma} n^{\delta}, \quad \Psi_{-1}=-C_{\alpha \beta \gamma \delta} l^{\alpha} n^{\beta} n^{\gamma} m^{* \delta}, \\
\Psi_{-2} & =-C_{\alpha \beta \gamma \delta} n^{\alpha} m^{* \beta} n^{\gamma} m^{* \delta} .
\end{aligned}
$$

Again, the subscript gives the spin weight and the conformal weight. (The notation here follows Price ${ }^{7}$ and differs somewhat from most authors.) For gravitational perturbations there is one additional complication: The null tetrad to be used in (2.11) is the NP special null tetrad associated with the perturbed metric, not the tetrad (2.8). The only $\Psi_{b}$ which is nonzero in the unperturbed Schwarzschild background is $\Psi_{0}=-M / r{ }^{3}$ The real parts of the $\Phi_{\phi}$ and $\Psi_{b}$ are associated with even-parity fields, and the imaginary parts are associated with odd-parity fields. The letter $p$ is used to denote the spin weight, since we reserve the letter $s$ for the spin of the field.

It is natural to take advantage of the spherical symmetry of the background to expand the perturbations in spherical harmonics. However, the appropriate spherical harmonics for the NP scalars with nonzero spin weight are not ordinary scalar spherical harmonics, but rather the spin-weight- $p$ spherical harmonics. ${ }^{12}$ These harmonics are denoted by ${ }_{p} Y_{m}^{l}(\theta, \varphi)$ and involve derivatives of the ordinary spherical harmonics, which have 
spin weight zero. The spin-weight index $p$ can be increased or decreased by certain differential operators. The spin-weight- $p$ spherical harmonics with $l<|p|$ are undefined.

The derivation of the equations governing scalar, electromagnetic, and gravitational test fields in the Schwarzschild background is described by Price. ${ }^{7} \mathrm{Be}-$ fore he expands in spherical harmonics, Price "despins" Newman-Penrose scalars with nonzero spin weight. This differs from conventional practice. 4,13 Therefore, we expand a Newman-Penrose scalar of spin weight $p$ directly in spin-weight-p spherical harmonics. For example,

$$
\Phi_{p}(u, r, \theta, \varphi)=\sum_{l=|p|}^{\infty} \Phi_{m}^{l}(u, r)_{p} Y_{m}^{l}(\theta, \varphi)
$$

To avoid an unnecessarily complicated notation we suppress the $l, m$ indices and write ${ }_{p} \Phi_{m}^{l}(u, r)$ as $\Phi_{p}(u, r)$. Since the equations for the different multipoles separate, this never causes any confusion.

The differential operators in the spin-coefficient formalism which contain derivatives with respect to $u$ and $r$ are

$$
D=l^{\mu} \frac{\partial}{\partial x^{\mu}}=\frac{\partial}{\partial r}
$$

and

$$
\Delta=n^{\mu} \frac{\partial}{\partial x^{\mu}}=\frac{\partial}{\partial u}-\frac{1}{2}\left(\frac{1-2 M}{r}\right) \frac{\partial}{\partial r}
$$

in the Schwarzschild background.

Of course, the spin-coefficient formalism is not needed for a scalar test field $\psi$, which satisfies

$$
\square \psi=(-g)^{-1 / 2} \frac{\partial}{\partial x^{\mu}}\left((-g)^{1 / 2} g^{\mu \nu} \frac{\partial \psi}{\partial x^{\nu}}\right)=0 .
$$

After expanding in ordinary scalar spherical harmonics, the equation for the $2^{l}$-pole is

$$
2 \frac{\partial^{2} \psi}{\partial r \partial u}+\frac{2}{r} \frac{\partial \psi}{\partial u}\left[\left(1-\frac{2 M}{r}\right) \frac{\partial \psi}{\partial r}\right]+\frac{l(l+1)}{r^{2}} \psi=0 .
$$

The equations governing the $2^{l}$-pole of an electromagnetic test field are

$$
\begin{gathered}
D\left[r^{2} \Phi_{0}\right]=\left[\frac{1}{2} l(l+1)\right]^{1 / 2} r \Phi_{1}, \\
D\left[r \Phi_{-1}\right]=\left[\frac{1}{2} l(l+1)\right]^{1 / 2} \Phi_{0}, \\
\Delta\left[(1-2 M / r) r \Phi_{1}\right] \\
=-\left[\frac{1}{2} l(l+1)\right]^{1 / 2}(1-2 M / r) \Phi_{0} \\
\Delta\left[r^{2} \Phi_{0}\right]=-\left[\frac{1}{2} l(l+1)\right]^{1 / 2} r \Phi_{-1} .
\end{gathered}
$$

These combine to give decoupled second-order differential equations for each of the $\Phi_{p}(u, r)$ :

$$
\begin{gathered}
\left.D\left\{(1-2 M / r)^{-1} r^{2} \Delta /(1-2 M / r) r \Phi_{1}\right]\right\} \\
+\frac{1}{2} l(l+1) r \Phi_{1}=0, \\
D \Delta\left[r^{2} \Phi_{0}\right]+\frac{1}{2} l(l+1) \Phi_{0}=0 \\
\Delta\left[r^{2} D\left(r \Phi_{-1}\right)\right]+\frac{1}{2} l(l+1) r \Phi_{-1}=0 .
\end{gathered}
$$

If any one of Eqs. (2.17) is solved, the corresponding solutions for the other two $\Phi_{b}$ are immediately obtained from Eqs. (2.16) as derivatives of the first $\Phi_{p}$. Price works with (2.17b); in Sec. 3 we solve Eqs. (2.17a) and (2.17c).

The equations for the gravitational test field are considerably more complicated, since the perturbations involve the very geometry through which the waves propagate. The spin coefficients $\rho, \lambda, \mu, \nu, \sigma, \tau$ and the metric functions $U, \omega$ appear in the equations for the $\Psi_{p}(u, r)$. In the equations governing a particular $2^{l}$-pole these subsidiary quantities, like the $\Psi_{p}$, are interpreted as the coefficients of the appropriate spin-weight spherical harmonics. The functions $\rho, \mu$, and $U$ have spin weight zero; $\tau$ and $\omega$ have spin weight $+1 ; \nu, \lambda$, and $\sigma$ have spin weights $-1,-2$, and +2 , respectively. For those quantities nonzero in the back ground we distinguish the perturbations by a subscript $B$.

The gravitational analogs of Eqs. (2.16) are the perturbed Bianchi identities:

$$
\begin{gathered}
D\left[r^{4} \Psi_{1}\right]=\left[\frac{1}{2}(l-1)(l+2)\right]^{1 / 2} r^{3} \Psi_{2} ; \\
D\left[r^{3} \Psi_{0_{B}}\right]=\left[\frac{1}{2} l(l+1)\right]^{1 / 2} r^{2} \Psi_{1}-3 M \rho_{B} ; \\
D\left[r^{2} \Psi_{-1}\right]=\left[\frac{1}{2} l(l+1)\right]^{1 / 2} r \Psi_{0_{B}}-3 M r^{-2} \omega^{*} ; \\
D\left[r \Psi_{-2}\right]=\left[\frac{1}{2}(l-1)(l+2)\right]^{1 / 2} \Psi_{-1}+3 M^{-2} \lambda ; \\
(1-2 M / r)^{-2} \Delta\left[(1-2 M / r)^{2} r \Psi_{2}\right] \\
=-\left[\frac{1}{2}(l-1)(l+2)\right]^{1 / 2} \Psi_{1}-3 M r^{-2} \sigma ; \\
(1-2 M / r)^{-1} \Delta\left[(1-2 M / r) r^{2} \Psi_{1}\right] \\
=-\left[\frac{1}{2} l(l+1)\right]^{1 / 2} r \Psi_{0}+3 M r^{-1}\left(\tau+r^{-1} \omega\right) ; \\
\Delta\left[r^{3} \Psi_{0}\right]=-\left[\frac{1}{2} l(l+1)\right]^{1 / 2} r^{2} \Psi_{-1} \\
+3 M\left(\mu_{B}-r^{-1} U_{B}\right) ; \\
(1-2 M / r) \Delta\left[(1-2 M / r)^{-1} r^{4} \Psi_{-1}\right] \\
=-\left[\frac{1}{2}(l-1)(l+2)\right]^{1 / 2} r^{3} \Psi_{-2}-3 M r \nu .
\end{gathered}
$$

The other equations 7,11 relating the metric perturbations, the perturbations in the spin coefficients, and the $\Psi_{p}$ are sufficiently complicated that it does not seem possible to combine them with Eqs. (2.18) to get a decoupled second-order differential equation for each of the $\Psi_{p}$. Price does derive such an equation for $\operatorname{Im} \Psi_{0_{B}}$.

However, Price turns to the Regge-Wheeler formalism, 14 as further developed by Zerilli, 15 to treat the evenparity gravitational perturbations.

Fortunately, decoupled equations do exist for $\Psi_{2}$ and $\Psi_{-2}$. The additional equations required are

$$
D\left(r^{2} \sigma\right)=r^{2} \Psi_{2}
$$

and

$$
\begin{aligned}
& (1-2 M / r) \Delta\left[(1-2 M / r)^{-1} r^{2} \lambda\right] \\
& =\left[\frac{1}{2}(l-1)(l+2)\right]^{1 / 2} r \nu-r^{2} \Psi_{-2} .
\end{aligned}
$$

Equations (2.18a), (2.18e), and (2.19) combine to give

$$
\begin{aligned}
& D\left\{(1-2 M / r)^{-2} r^{4} \Delta\left[(1-2 M / r)^{2} r \Psi_{2}\right]\right\} \\
& +\left[\frac{1}{2}(l-1)(l+2)+3 M / r\right] r^{3} \Psi_{2}=0,
\end{aligned}
$$


while Eqs. (2.18d), (2.18h), and (2.20) give

$$
\begin{aligned}
& \Delta\left\{(1-2 M / r)^{-1} r^{4} D\left[r \Psi_{-2}\right]\right\} \\
& +\left[\frac{1}{2}(l-1)(l+2)+3 M / r\right](1-2 M / r)^{-1} r^{3} \Psi_{-2}=0 .
\end{aligned}
$$

The outgoing radiation field near future null infinity is contained in $\Psi_{-2}$ and the Newman-Penrose quantities are in $\Psi_{2}$; so, for our purposes a complete solution for all of the spin coefficients and the remianing $\Psi_{p}$ is not necessary.

We shall see below that Eqs. (2.15), (2.17a), and (2.17c), for scalar and electromagnetic test fields, are all special cases of a single "master" equation. It is a rather remarkable fact that Eqs. (2.21a) and (2.21b), which govern the radiative behavior of gravitational test fields, are also special cases of the same equation. In this sense the Einstein field equations, with their particular coupling of the perturbations to the background geometry, represent the simplest spin-2 field equations in the curved Schwarzschild background. The solutions to the master equation governing all the fields depend explicitly on $s$ (the spin of the perturbing field) only in a very minimal way.

\section{THE GENERAL RETARDED SOLUTION}

We consider the equation

$$
\begin{aligned}
& 2 \frac{\partial^{2 \psi}}{\partial u \partial r}+\frac{2(s+p+1)}{r} \frac{\partial \psi}{\partial u} \\
& \quad-\frac{\partial^{2} \psi}{\partial r^{2}}-\frac{(2 s+2)}{r} \frac{\partial \psi}{\partial r}+\frac{(l+s+1)(l-s)}{r^{2}} \psi \\
& \quad+\left(\frac{2 M}{r}\right)\left(\frac{\partial^{2} \psi}{\partial r^{2}}+\frac{(2 s+1-p)}{r} \frac{\partial \psi}{\partial r}+\frac{s(s-p)}{r^{2}} \psi\right)=0
\end{aligned}
$$

The parameter $s$ takes on the values 0,1 , or 2 corresponding to the spin of the test field. The parameter $p$ takes on the values $\pm s$ corresponding to the two extreme possible spin weights. [Eq. (3.1) can not be used for "nonradiative" spin-weight components $-s+1 \leq p \leq$ $s-1$.] With $s=0, p=0, \psi(u, r)$ is the coefficient of $Y_{m}^{l}(\theta, \varphi)$ in the spherical harmonic expansion of a scalar test field, and Eq. (3.1) is identical to the field equation (2.15). With $s=1, p= \pm 1, \psi$ represents the coefficient of ${ }_{1}^{l}$ in the spin weight \pm 1 part of the electromagnetic field tensor; (3.1) then is identical to (2.17a) and $(2.17 \mathrm{c})$. With $s=2, p= \pm 2, \psi$ represents the coefficient of ${ }_{ \pm 2} Y^{l}$ in the spin weight \pm 2 part of the Weyl tensor, and (3.1) becomes identical to (2.21a) and (2.21b). In this section we obtain a general retarded solution to this master equation.

The solution is in the form of an expansion in powers of the gravitational mass $M$. We prove that the expansion converges for retarded fields at all $r>R>2 M$, where $R$ is a radius bounding both the source of the background Schwarzschild metric (either a star or a black hole) and the source of the test field at all times to the past.

The general retarded solution to Eq. (3.1) must be regular at infinity in the minimal sense that $\psi \rightarrow 0$ as $r \rightarrow \infty$, and must be entirely generated by sources in the region $r<R$. It will contain one arbitrary function of the retarded time $u$.

First consider static solutions. Since $r^{-1}=0$ is a regular singular point of the ordinary differential equation for $\psi(r)$, the static solution regular at infinity can be written as the series

$$
\psi(r)=A 2^{(p-s) / 2} r^{-(l+s+1)}\left[1+\sum_{k=1}^{\infty} a_{k}\left(\frac{2 M}{r}\right) k\right]
$$

with

$$
a_{k}=\frac{(l+k) !}{l ! k !} \frac{(l+p+k) !}{(l+p) !} \frac{(2 l+1) !}{(2 l+1+k) !} .
$$

The series converges for all $r>2 M$. The coefficient $A$ is identified as the static multipole moment.

Now consider solutions to Eq. (3.1) which are static for all $u \leq u_{0}$, but dynamic for $u>u_{0}$. These solutions are retarded, since no incoming waves are present near past null infinity. At $u=u_{0}, \psi$ can be expanded in powers of $r^{-1}$ at fixed $u$, and this analytic structure will persist for a finite retarded time after $u=u_{0}$ in the region $r>R$. Let

$$
\psi=\sum_{n} f_{n}(u) r^{-n}
$$

and substitute into Eq. (3.1). The $f_{n}$ must satisfy the hierarchy of equations.

$$
\begin{aligned}
& 2(n-p-s-1) f_{n}{ }^{\prime}=(l+s+2-n)(n+l-s-1) f_{n-1} \\
& \quad+(2 M)(n+p-s-2)(n-s-2) f_{n-2} .
\end{aligned}
$$

It is consistent with Eq. (3.5) that all $f_{n}$ with $n<p+s+$ 1 are identically zero. Furthermore, these $f_{n}$ must be zero, or some $f_{n}$ with $n \leq 0$ will be nonzero, and $\psi(u, r)$ will not go to zero as $r \rightarrow \infty$ at $u>u_{0}$. This is the peeling property at future null infinity.

Split the sum (3.4) into two parts:

and

$$
\psi_{\mathrm{I}}=\sum_{n=p+s+1}^{l+s+1} f_{n}(u) r^{-n}
$$

$$
\psi_{\mathrm{II}}=\sum_{n=l+s+2}^{\infty} f_{n}(u) r^{-n}
$$

We shall see that the $f_{n}$ in $\psi_{1}$ are linear combinations of a single function of retarded time, the retarded multipole moment $A(u)$, and its first $(l-p)$ derivatives. The $f_{n}$ in $\psi_{\text {II }}$ cannot be represented in this way consistent with Eqs. (3.5) and the static initial conditions.

Define the multipole moment $A(u)$ from the part of the field with $p=s$. Let

$f_{2 s+1}(u)=\lim _{r \rightarrow \infty}\left[r^{2 s+1} \psi(u, r)\right]=\frac{2^{l-s}(l+s) !}{(2 l) !} A^{(l-s)}(u)$.

(Superscripts in parentheses denote the number of derivatives to be taken.) In the first $(l-s)$ successive integrations of Eqs. (3.5), with $p=s$, absorb the constants of integration into $A(u)$. Then $f_{l+s+1}=A$ when $A(u)$ is constant, consistent with Eq. $(3.2)$.

The coefficient of $r^{-1}, f_{1}(u)$, in the $\psi(u, r)$ with $p=-s$ is related to $f_{2+1}(u)$ in the $\psi(u, r)$ with $p=s$ by the flat-space versions of Eqs. $(2,16 \mathrm{c})$ and $(2.16 \mathrm{~d})$ coupling the $\Phi_{p}(s=1)$ and Eqs. $(2.18 \mathrm{e})-(2.18 \mathrm{~h})$ coupling the $\Psi_{p}$ $(s=2)$. The terms in these equations from the curvature of the background are of order $r^{-1}$; at least as long as the infinite sum in $\psi_{\mathrm{II}}$ converges, one has

$$
\frac{\partial \psi}{\partial r}=\theta\left(r^{-1} \psi\right)
$$


just as for the flat-space retarded solutions. Therefore, the analog of Eq. (3.8) when $p=-s$ is

$$
\begin{aligned}
\left.f_{1}(u)\right|_{p=-s}=\left.2 s \frac{(l-s) !}{(l+s) !} f_{2 s+1}(2 s)\right|_{p=s} \\
=2 l \frac{(l-s) !}{(2 l) !} A^{(l+s)}(u) .
\end{aligned}
$$

Starting with either Eq. (3.8) or Eq. (3.10), successive integrations of Eqs. (3.5) give the $f_{n}(u)$ in $\psi_{\mathrm{I}}(u, r)$ in the form

$$
f_{p+s+1+k}=\sum_{m=0}^{[k / 2]} \alpha_{k, m}(2 M)^{m} A^{(l-p-k+m)} .
$$

The upper limit to the sum $[k / 2]$ is

$$
[k / 2]=\left\{\begin{array}{ll}
k / 2, & k \text { even } \\
(k-1) / 2, & k \text { odd }
\end{array} .\right.
$$

All the coefficients $\alpha_{k, m}$ can easily be evaluated for any particular values of $s, l$, and $p$ [see Eq. (4.19) and following, for an example]; the coefficients which survive when $M=0$ are

$$
\alpha_{k, 0}=2^{l-k} 2^{-(p+s) / 2} \frac{(l+p+k) !}{(2 l) !} \frac{(l-p) !}{k !(l-p-k) !} .
$$

When the field is static, the only nonzero $f_{n}$ in $\psi_{\mathrm{I}}$ is

$$
f_{l+s+1}=2^{(p-s) / 2} A \text {. }
$$

Because the coefficient of $f_{n-1}$ in Eq. (3.5) vanishes when $n=l+s+2$, the constant of integration in $f_{l+s+2}$ cannot be absorbed in $\int^{\mu} A\left(u^{\prime}\right) d u^{\prime}$ as, for instance, the constant of integration in $f_{l+s+1}$ was absorbed in $A(u)$. Instead, it must be kept explicitly:

$f_{l+s+2}=C+\frac{l(l+p)}{2(l-p+1)} \sum_{m=0}^{[(l-p-1) / 2]} \alpha_{l-p-1, m}(2 M)^{m+1} A^{(m)}$.

From the static initial condition on $f_{l+s+2}$,

$$
C=\frac{1}{2} 2^{(p-s) / 2} \frac{(2 l+1)}{l-p+1}(2 M) A\left(u_{0}\right) .
$$

The fact that the constant of integration contains $A\left(u_{0}\right)$ means that $f_{l+s+2}(u)$ for $u>u_{0}$ depends on the past history of the time dependence of $A(u)$, as well as on the instantaneous values of $A(u)$ and its derivatives. The backscatter of the outgoing radiation field $\psi_{I}(u, r)$ is entirely contained in $\psi_{i}(u, r)$. It was the failure to allow for the constant of integration (3.16) that led to the incorrect treatment of the backscatter in preprint versions of papers by Price ${ }^{7}$ and Thorne ${ }^{16}$ on the decay of radiatable multipoles during gravitational collapse.

The integration constant in $f_{l+s+2}$ generates terms in the $f_{n}$ with $n>l+s+2$ which grow with time:

$$
\begin{aligned}
f_{l+s+1+k} \sim(-1)^{k-1} & \frac{(k-1) !}{2^{k}} \frac{(l-p) !}{(l-p+k) !} \\
& \times \frac{(2 l+k) !}{(2 l) !} 2^{(p-s) / 2}(2 M) A\left(u_{0}\right) u^{k-1} .
\end{aligned}
$$

While these terms may be partially cancelled by terms coming from successive integrals of $A(u)$, typically

$$
\frac{f_{l+s+k+1}}{f_{l+s+k}} \sim-\frac{u-u_{0}}{2}
$$

in the limit $k \gg l$ when $u-u_{0} \gg 2 M$, so the expansion

(3.7) of $\psi_{\text {II }}$ in powers of $r^{-1}$ will diverge once

$$
u-u_{0}>2 r \text {. }
$$

Thus, the power series expansion of the form (3.4) is not a satisfactory solution of Eq. (3.1). ${ }^{3}$

To obtain a solution which converges uniformly at all future times, we keep $\Psi_{I}$ in the form $(3.6)$, but represent $\psi_{\mathrm{II}}(u, r)$ by

$\psi_{\mathrm{II}}(u, r)=r^{-(l+s+1)} \sum_{k=1}^{\infty} a_{k}\left(\frac{2 M}{r}\right)^{k} g_{k}(u, r)$.

This new expansion is an expansion in powers of the gravitational mass $M$, instead of powers of $r^{-1}$. The coefficients $a_{k}$ are the coefficients (3.3) in the static solution. For the purposes of the new expansion $\psi_{\mathrm{I}}(u, r)$ is considered zeroth order in $M$, even though $M$ appears in the $f_{n}(u), n \leq l+s+1$, through Eq. (3.11).

Substitute the expression (3.20) into Eq. (3.1), along with $\psi_{\mathrm{I}}(u, r)$ in the form $(3.6)$, and require that the coefficent of each explicit power of $M$ vanish. The result is a hierarchy of partial differential equations for the $g_{k}(u, r)$ : When $k>1$,

$$
\begin{aligned}
2 \frac{\partial^{2} g_{k}}{\partial u \partial r}- & \frac{2(k+l-p)}{r} \frac{\partial g_{k}}{\partial u}-\frac{\partial^{2} g_{k}}{\partial r^{2}} \\
& +\frac{2(k+l)}{r} \frac{\partial g_{k}}{\partial r}-\frac{k(k+2 l+1)}{r^{2}} g_{k} \\
& =-\frac{k(k+2 l+1)}{(k+l)(k+l+p)}\left(\frac{\partial^{2} g_{k-1}}{\partial r^{2}}-\frac{(2 k+2 l+p-1)}{r} \frac{\partial g_{k-1}}{\partial r}\right. \\
& \left.+\frac{(k+l)(k+l+p)}{r^{2}} g_{k-1}\right) ;
\end{aligned}
$$

and when $k=1$,

$$
\begin{aligned}
2 \frac{\partial^{2} g_{1}}{\partial u \partial r}- & \frac{2(l-p+1)}{r} \frac{\partial g_{1}}{\partial u}-\frac{\partial^{2} g_{1}}{\partial r^{2}} \\
& +\frac{2(l+1)}{r} \frac{\partial g_{1}}{\partial r}-\frac{(2 l+2)}{r^{2}} g_{1} \\
= & -\frac{(2 l+2)}{r^{2}} f_{l+s+1}(u)-\frac{2 l(l+p)}{l+p+1} \frac{f_{l+s}(u)}{r} .
\end{aligned}
$$

The right-hand side of Eq. (3.22) comes from using Eqs. (3.5) on the $f_{n}$ in $\psi_{\mathrm{I}}$.

Equations (3.21) are scale invariant under the transformation $u \rightarrow K u, r \rightarrow K r$. Equation (3.22) is not generally scale invariant; but it is if the multipole moment $A(u)$ is constant, which implies that $f_{l+s+1}$ is constant and $f_{l+s}$ is zero. In this special case the entire hierarchy of equations for the $g_{k}$ is invariant under the scale transformation. The scale invariance suggests that a solution to the hierarchy exists which depends on only one independent variable, a scale invariant combination of $u$ and $r$. Since the equations are also invariant under a translation in $u$ when $A(u)$ is constant, the most general form for the similarity variable is

$$
y=\left(u-u_{1}\right) / 2 r .
$$


The similarity solutions will be superimposed to give the general retarded solution.

The ansatz $g_{k}(u, r)=g_{k}(y)$ reduces the partial differential equations $(3,21)$ and $(3,22)$ to the ordinary differential equations

$$
\begin{aligned}
y(1+ & +y) \frac{d^{2} g_{k}}{d y^{2}}+[k+l-p+1+2(k+l+1) y] \frac{d g_{k}}{d y} \\
& +k(k+2 l+1) g_{k}=\frac{k(k+2 l+1)}{(k+l)(k+l+p)}\left(y^{2} \frac{d^{2} g_{k-1}}{d y^{2}}\right. \\
& \left.+(2 k+2 l+p+1) y \frac{d g_{k-1}}{d y}+(k+l)(k+l+p) g_{k-1}\right) .
\end{aligned}
$$

When $k=1$ in Eq. $(3.24)$ the $g_{0}$ appearing on the righthand side is understood to be $f_{l+s+1}$, a constant.

The solution of Eqs. $(3,24)$ can be reduced to quadratures by standard methods. A particular solution to the hierarchy is the solution, for which

$$
g_{k}(y)=f_{l+s+1}=2^{(p-s) / 2} A,
$$

for all $k \geq 1$. Any dynamic solution to the hierarchy is a particular solution plus a homogeneous solution. To join a dynamic solution to a static solution at $u=u_{0}$ it is necessary to take $u_{1}=u_{0}$, or

$$
y=\left(u-u_{0}\right) / 2 r,
$$

since only at $y=0$ is $y$ independent of $r$ at fixed $u$. Therefore, the homogeneous solutions for constructing initially static dynamic solutions must be regular at $y=0$.

Only one of the two independent homogeneous solutions to the $k$ th equation $(3.24)$ is regular at $y=0$. Normalized to be one at $y=0$, it is

$$
\begin{aligned}
h_{k}(y)=(1+y)^{-(k+l+p)} \sum_{m=0}^{l+p} \frac{(l+p) !}{m !(l+p-m) !} \\
\quad \times \frac{(l-p+k) !}{l-p+k+m) !} \frac{(l-p+m) !}{(l-p) !} y^{m} .
\end{aligned}
$$

A homogeneous solution to the hierarchy is composed of inhomogeneous solutions to Eq. (3.24) for all $k>n$, generated by the homogeneous solution $(3.27)$ for $k=n$. Let the functions $H_{n, k}(y)$ be the $g_{k}(y)$ generated by $h_{n}(y)$ :

$$
g_{k}(y)=H_{n, k}(y) \text {. }
$$

For $k<n$;

$$
H_{n, k}=0 \text {. }
$$

The nonzero $H_{n, k}$ are all normalized to be one at $y=0$. Thus

$$
H_{n, n}(y)=h_{n}(y)
$$

and for $k>n$

$H_{n, k}(y)=h_{k}(y)\left(1+\int_{0}^{y} d y_{1} y_{1}-(k+l-p+1)\left(1+y_{1}\right)^{-(k+l+p+1)}\right.$

$\left.\times h_{k}\left(y_{1}\right)^{-2} \int_{0}^{y_{1}} d y_{2} y_{2}{ }^{k+l-p}\left(1+y_{2}\right)^{k+l-p h_{k}}\left(y_{2}\right) S_{k}\left(y_{2}\right)\right) \cdot(3.31)$

The function $S_{k}$ is the right-hand side of the $k$ th equation
(3.24), with $g_{k-1}=H_{n, k-1}$.

Some important properties of the nonzero $H_{n, k}$ are

$$
H_{n, k}=1-\theta\left(y^{k-n+1}\right)
$$

in the limit $y \ll 1$, while when $y \gg 1$

$$
\begin{aligned}
H_{n, k} & \simeq \frac{k !}{(k-n) !} \frac{(k+2 l+1) !}{(k+2 l+1+n) !} \frac{(k+l-n) !}{(k+l) !} \\
& \times \frac{(k+l+p-n) !}{(k+l+p) !} \frac{(l+n) !}{l ! n !} \frac{(l-p+n) !}{(l-p) !} \frac{(l+p+n) !}{(l+p) !} \\
& \times \frac{(2 l) !}{(2 l+n) !} \frac{(2 l+1) !}{(2 l+n+1) !} y^{-n} .
\end{aligned}
$$

The nonzero $H_{n, k}$ decreases monotonically from one at $y=0$ to zero in the limit $y \rightarrow \infty$.

The leading term in a homogeneous solution $H_{n, n}(y)$ can be interpreted as an ingoing wave in flat space. That is,

$$
\psi=h_{n}(y) r^{-(l+s+1+n)}
$$

solves Eq. (3.1) with $M=0$ and can be put in the form

$$
\begin{aligned}
\psi=\sum_{q=0}^{l+p} 2^{-(s+p) / 2} & (-2) q \frac{(2 l-q) !}{(2 l) !} \\
& \times \frac{(l+p) !}{q !(l+p-q) !} B(q)(v) r^{-(l+s+1-q) .}
\end{aligned}
$$

The advanced multipole moment $B(v)$, as a function of the flat-space advanced time

is

$$
v=u+2 r \text {, }
$$

$$
B(v)=2^{(s+p) / 2} \frac{(2 l) !}{(2 l+n) !} \frac{(l-p+n) !}{(l-p) !} 2^{n}\left(v-u_{0}\right)^{-n} .
$$

A similarity solution solves the following problem: The field is static for all $u<u_{0}$; at $u=u_{0}$ the retarded multipole moment changes by a step function to a new constant value for all $u>u_{0}$. With the help of the above homogeneous solutions it is possible to fit the initial conditions on the $g_{k}(y)$ at $u$ infinitesimally greater than $u_{0}$. The instantaneous changes in the $g_{k}$ due to the change in $A(u)$ are found from Eqs. (3.5).

An arbitrary continuous variation of $A(u)$ can be approximated arbitrarily closely by a superposition of stepfunction changes. Since the test-field equations are linear, the general retarded solution to Eq. (3.1) can be represented as a continuous superposition of similarity solutions. The constant $u_{0}$ in the similarity variable $y$ becomes a dummy integration variable. By letting the range of integration extend to $u_{0}=-\infty$, we include cases in which the field was never static at any time in the past.

Our general retarded solution for $g_{k}(u, r)$ is

$$
\begin{aligned}
g_{k}(u, r) & =f_{l+s+1}(u)-\int_{-\infty}^{u} d u_{0}\left[\left(\frac{d f_{l+s+1}}{d u_{0}}\right.\right. \\
- & \left.\frac{l(l+p)}{(l-p+1)(l+p+1)} f_{l+s}\left(u_{0}\right)\right) H_{1, k}(y) \\
+ & \left.\frac{l(l+p)}{(l-p+1)(l+p+1)} f_{l+s}\left(u_{0}\right) H_{2, k}(y)\right] .
\end{aligned}
$$


That this does indeed solve Eqs. (3.21) and (3.22) can easily be checked by substitution. The integral in Eq. (3.38) is the backscatter; when $k=1$ it is a superposition of purely ingoing waves generated by previous changes in the multipole moment.

While Eq. (3.38) is best for the physical interpretation of $g_{k}(u, r)$, a different form of the solution is best for proving convergence of the integrals and of the series (3.20). Integrate by parts in Eq. (3.38) and define

$$
\begin{gathered}
F_{l+s}(u) \equiv \frac{l(l+p)}{(l-p+1)(l+p+1)} \int^{u} f_{l+s}\left(u_{0}\right) d u_{0} \\
\equiv \frac{l(l+p)}{(l-p+1)(l+p+1)} \\
\quad \times \sum_{m=0}^{[(l-p-1) / 2]} \alpha_{l-p-1, m}(2 M)^{m} A(m)(u) .
\end{gathered}
$$

When $r$ is finite, so that $u_{0} \rightarrow-\infty$ implies $y \rightarrow \infty$, the result is

$$
\begin{aligned}
g_{k}(u, r)= & F_{l+s}(u) \delta_{k 1} \\
& -\frac{1}{2 r} \int_{-\infty}^{u} d u_{0}\left\{\left[f_{l+s+1}\left(u_{0}\right)-F_{l+s}\left(u_{0}\right)\right] H_{1, k}(y)\right. \\
& \left.+F_{l+s}\left(u_{0}\right) H_{2, k}(y)\right\}
\end{aligned}
$$

The primes denote derivatives with respect to $y$, and $\delta_{k l}$ is the Kronecker delta.

In going from Eq. (3.38) to Eq. (3.40) we have implicitly assumed that $f_{l+s+1}(u)$ and $F_{l+s}(u)$ are bounded in the limit $u \rightarrow-\infty$. We now impose the slightly stronger condition that $f_{l+s+1}\left(u_{0}\right)$ and $F_{l+s}\left(u_{0}\right)$ be bounded for all $u_{0}<u$, if the field is being evaluated at the retarded time $u$. Both $f_{l+s+1}\left(u_{0}\right)$ and $F_{l+s}\left(u_{0}\right)$ contain at most $(l-p)$ derivatives of $A\left(u_{0}\right)$, so the condition follows if $\psi_{\mathrm{I}}\left(u_{0}, r\right)$ was bounded at all times to the past.

Since the $H_{n, k}$ decrease monotonically from one at $y=0$ to zero at $y=\infty$, the $H_{n, k}{ }^{\prime}(y)$ in Eq. (3.40) are negative or zero over the whole range of integration. If $f_{l+s+1}\left(u_{0}\right)$ and $F_{l+s}\left(u_{0}\right)$ satisfy

$$
\begin{aligned}
& \left|f_{l+s+1}\left(u_{0}\right)\right| \leq K_{1}, \\
& \left|F_{l+s}\left(u_{0}\right)\right| \leq K_{2}
\end{aligned}
$$

for all $-\infty<u_{0} \leq u$, then

$$
\begin{aligned}
& -K_{1} \leq-\frac{1}{2 r} \int_{-\infty}^{u} d u_{0} f_{l+s+1}\left(u_{0}\right) H_{1, k}{ }^{\prime}(y) \\
& \leq-\frac{K_{1}}{2 r} \int_{-\infty}^{u} d u_{0} H_{1, k}{ }^{\prime}(y)=K_{1}, \\
& -K_{2} \leq-\frac{1}{2 r} \int_{-\infty}^{u} d u_{0} F_{l+s}\left(u_{0}\right) H_{n, k}{ }^{\prime}(y) \leq K_{2} \text {, } \\
& \left|g_{k}(u, r)\right| \leq K_{1}+2 K_{2} .
\end{aligned}
$$

Both $K_{1}$ and $K_{2}$ are the same order as the bound on $\left|A\left(u_{0}\right)\right|$, since the time scale over which $A(u)$ changes is typically greater than or equal to $(2 M)$.

The integrals in Eq. (3.38) will not necessarily converge to any definite value at $r=\infty$, where $y=0$ for all $u_{0}$. Since $r=\infty$ is not in the physical space-time, there is not physical requirement that the $g_{k}(u, \infty)$ have welldefined values.

Derivatives of the $g_{k}$ with respect to $u$ and $r$ do not affect the convergence of the integrals, since

$$
\frac{\partial}{\partial u} H_{n, k}(y)=\frac{1}{2 r} H_{n, k}{ }^{\prime}(y) \sim \frac{1}{r} y^{-(n+1)}
$$

and

$$
\frac{\partial}{\partial r} H_{n, k}(y)=-\frac{y}{r} H_{n, k}{ }^{\prime}(y) \sim \frac{1}{r} y^{-n},
$$

$y \gg 1$. Equation (3.9) is valid for the general solution, not only initially static solutions.

From Eq. (3. 45), the absolute values of the $g_{k}(u, r)$ are bounded uniformly in $k$. We conclude that the infinite sum in Eq. (3.20) for $\psi_{\mathrm{II}}(u, r)$ is absoutely convergent at all $r>R$, for any $R>2 M$, and that

$$
\psi_{\text {II }}(u, r)=\theta\left(r^{-(l+s+2))} .\right.
$$

The only restrictions on the time dependence of the retarded multipole moment $A(u)$ are boundedness condi tions on $A(u)$ and its first $[(l-p) / 2]$ derivatives. These are physically necessary conditions if the field is to have finite energy density at all times to the past. Our general retarded solution constructed from Eqs. (3.6) and (3.20), with the $f_{n}(u)$ given by Eq. (3.11) and the $g_{k}(u, r)$ given by Eq. (3.38) or (3.40), almost certainly contains all physically nonsingular retarded solutions to Eq. (3.1).

Some results of this section are not new. The solutions of Couch et al.17,18 for the backscatter of electromagnetic and gravitational radiation first order in $M$ are essentially the same as Eq. (3.40), with $k=1$.

\section{THE LOWEST RADIATABLE MULTIPOLES}

The physically most important multipoles are the electromagnetic dipole and the gravitational quadrupole. These typically dominate in electromagnetic and gravitational radiation processes, respectively. They are the lowest multipoles which can radiate, i.e., contribute $r^{-1}$ terms in the respective field tensors at future null infinity. Furthermore, these multipoles contain the apparently conserved Newman-Penrose quantities. In this section we write out explicitly the general retarded solutions for $\Phi_{ \pm 1}$ (electromagnetic) and $\Psi_{ \pm 2}$ (gravitational) through order $(2 \mathrm{M} / r)$ in all cases and through order $(2 \mathrm{M} / \mathrm{r})^{2}$ for $\Psi_{-2}$. Higher-order terms do not contribute to the NPQ's.

\section{A. The electromagnetic dipole field}

In view of Eq. (3.8) the retarded electromagnetic dipole moment $E(u)$ is defined by

$$
E(u) \equiv \lim _{r \rightarrow \infty}\left[r^{3} \Phi_{1}(u, r)\right]
$$

in the dipole part of the field. We have shown in Sec. 3 that this limit always exists for retarded fields.

In the spin-weight-one part of the dipole field the function $h_{1}(y)$ is

$$
h_{1}(y)=\left(1+y+\frac{1}{3} y^{2}\right) /(1+y)^{3},
$$

so that

$$
g_{1}(u, r)=E(u)-\int_{-\infty}^{u} d u_{0} \frac{d E}{d u_{0}} \frac{1+y+\frac{1}{3} y^{2}}{(1+y)^{3}}
$$

with $y=\left(u-u_{0}\right) / 2 r$, or

$$
g_{1}(u, r)=\int_{0}^{\infty} d y E\left(u_{0}\right) \frac{2+\frac{4}{3} y+\frac{1}{3} y^{2}}{(1+y)^{4}}
$$


with

$$
u_{0}=u-2 r y \text {. }
$$

The result for $\Phi_{1}(u, r)$ is

$$
\begin{aligned}
& \Phi_{1}(u, r)=r^{-3} E(u)+\frac{3}{2}(2 M) r^{-4} \int_{0}^{\infty} d y E\left(u_{0}\right) \frac{2+\frac{4}{3} y+\frac{1}{3} y^{2}}{(1+y)^{4}} \\
& \quad+\Theta\left[(2 M / r)^{2}\right] .
\end{aligned}
$$

Alternatively, we could have begun with the spin-weightminus-one part of the dipole field. Here Eq. $(3.10)$ gives

$$
f_{1}(u)=E^{(2)}(u) \text {. }
$$

Applying Eq. (3.5) twice,

$$
f_{2}(u)=E^{(1)}(u)
$$

and

$$
f_{3}(u)=f_{l+s+1}(u)=\frac{1}{2} E(u) .
$$

The function $h_{1}(y)$ is simply

so

$$
h_{1}(y)=(1+y)^{-1}
$$

$$
\begin{aligned}
& g_{1}(u, r)=\frac{1}{2} E(u)-\int_{-\infty}^{u} d u_{0} \frac{1}{2} \frac{d E}{d u_{0}} \frac{1}{1+y} \\
& =\frac{1}{2} \int_{0}^{\infty} d y E\left(u_{0}\right)(1+y)^{-2} .
\end{aligned}
$$

In Eq. (4.11), as in Eq. (4. 3b), $u_{0}$ is given by Eq. (4.4). Putting everything together, we obtain

$$
\begin{aligned}
& \Phi_{1}(u, r)=r^{-1} E^{(2)}(u)+r^{-2} E^{(1)}(u)+\frac{1}{2} r^{-3} E(u) \\
& +\frac{1}{4}(2 M) r^{-4} \int_{0}^{\infty} d y E\left(u_{0}\right)(1+y)^{-2} \\
& +O\left[(2 M / r)^{2}\right] .
\end{aligned}
$$

For a given time dependence of the dipole moment $E(u)$, the solution (4.12) for $\Phi_{-1}$ must be consistent with the solution (4.5) for $\Phi_{1}$. This is easily checked by applying Eqs. (2.16a) and (2.16b) to the solution (4.12). First,

$$
\begin{aligned}
& \Phi_{0}=\frac{\partial}{\partial r}\left[r \Phi_{-1}\right] \\
& =-r^{2} E^{(1)}(u)-r^{-3} E(u) \\
& -(2 M) r^{-4} \int_{0}^{\infty} d y E\left(u_{0}\right) \frac{1+\frac{1}{2} y}{(1+y)^{3}} \\
& -\theta\left[(2 M / r)^{2}\right] .
\end{aligned}
$$

Then

$$
\Phi_{1}=r^{-1} \frac{\partial}{\partial r}\left[r^{2} \Phi_{0}\right]
$$

gives Eq. (4.5).

\section{B. The gravitational quadrupole field}

The gravitational quadrupole moment $G(u)$ is also very simply defined by Eq. (3.8):

$$
G(u) \equiv \lim _{r \rightarrow \infty}\left[r^{5} \Psi_{2}(u, r)\right] .
$$

The limit is again guaranteed to exist for retarded fields.

The analogs of Eqs. (4.2), (4.3a), (4.3b), and (4.5) for the spin-weight-two part of the gravitational quadrupole field are

$$
\begin{aligned}
& h_{1}(y)=\frac{1+2 y+2 y^{2}+y^{3}+\frac{1}{5} y^{4}}{(1+y)^{5}} \\
& g_{1}(u, r)=G(u)-\int_{-\infty}^{0} d u_{0} \frac{d G}{d u_{0}} \frac{1+2 y+2 y^{2}+y^{3}+\frac{1}{5} y^{4}}{(1+y)^{5}} \\
& =\int_{0}^{\infty} d y G\left(u_{0}\right) \frac{3+4 y+3 y^{2}+\frac{6}{5} y^{3}+\frac{1}{5} y^{4}}{(1+y)^{6}} \\
& \Psi_{2}(u, r)=r^{-5} G(u) \\
& +\frac{5}{2}(2 M) r^{-6} \int_{0}^{\infty} d y G\left(u_{0}\right) \frac{3+4 y+3 y^{2}+\frac{6}{5} y^{3}+\frac{1}{5} y^{4}}{(1+y)^{6}} \\
& +O\left[(2 M / r)^{2}\right]
\end{aligned}
$$

The spin-weight-minus-two part of the gravitational quadrupole field shows how the effects of the background curvature can enter $\psi_{[}(u, r)$. [See discussion following Eq. (3.10)] Start with

$$
f_{1}(u)=\frac{1}{6} G^{(4)}(u) .
$$

The successive integrations of Eq. (3.5) give

$$
\begin{aligned}
& f_{2}(u)=\frac{1}{3} G^{(3)}(u), \\
& f_{3}(u)=\frac{1}{2} G^{(2)}(u)+\frac{1}{8}(2 M) G^{(3)}(u), \\
& f_{4}(u)=\frac{1}{2} G^{(1)}(u)+\frac{1}{8}(2 M) G^{(2)}(u), \\
& f_{5}(u)=f_{l+s+1}(u)=\frac{1}{4} G(u)-\frac{1}{64}(2 M)^{2} G^{(2)}(u) .
\end{aligned}
$$

Similar curvature terms appear in $\Psi_{2}$ when $l \geq 4$ and in $\Phi_{1}$ when $l \geq 3$. Note that the $f_{n}(u), n<5$, cannot be expressed as a finite sum over derivatives of $f_{5}(u)$. For this reason, the quadrupole moment should not be defined as the coefficient of $r^{-5}$ in $\Psi_{-2}(u, r)$; rather, Eq. (4,15)which leads to Eq. (4.19)-is the better definition.

In $\Psi_{\mathrm{II}}(u, r)$ for $\Psi_{-2}$ the function $h_{1}(y)$ is again simple,

$$
h_{1}(y)=(1+y)^{-1} \text {. }
$$

This makes it feasible to go on and solve for $H_{1,2}(y)$, which appears in the solution for $g_{2}(u, r)$. The result of applying Eq. (3.31) is

$$
\begin{aligned}
& H_{1,2}(y)=(1+y)^{-2}\left\{1+\frac{7}{8}\left[y+\ln (1+y)+\frac{11}{30}\right.\right. \\
& -\frac{1}{2} y^{-1}+\frac{3}{4} y^{-2}-\frac{4}{3} y^{-3}+\frac{7}{2} y^{-4}+5 y^{-5} \\
& \left.\left.-(5+6 y) y^{-6} \ln (1+y)\right]\right\} .
\end{aligned}
$$

We finally have for $\Psi_{-2}$ :

$$
\begin{aligned}
& \Psi_{-2}(u, r)=\frac{1}{6} \gamma^{-1} G^{(4)}+\frac{1}{3} r^{-2} G^{(3)}+\frac{1}{2} \gamma^{-3}\left[G(2)+\frac{1}{4}(2 M) G^{(3)}\right] \\
& +\frac{1}{2} \gamma^{-4}\left[G(1)+\frac{1}{4}(2 M) G^{(2)}\right]+\frac{1}{4} r^{-5}\left[G-\frac{1}{16}(2 M)^{2} G^{(2)}\right] \\
& +\frac{1}{8} r^{-5}(2 M / r) \int_{0}^{\infty} d y\left[G\left(u_{0}\right)-\frac{1}{16}(2 M)^{2} G^{(2)}\left(u_{0}\right)\right](1+y)^{-2} \\
& +\frac{1}{14} r^{-5}(2 M / r)^{2} \int_{0}^{\infty} d y\left[G\left(u_{0}\right)-\frac{1}{16}(2 M)^{2} G^{(2)}\left(u_{0}\right)\right]\left[-H_{1,2}(y)\right] \\
& \quad+O\left[(2 M / r)^{3}\right] .
\end{aligned}
$$

The coefficient of $f_{l+s}$ vanishes in Eqs. (3.38)-(3.40) when $l=-p=s$, which means that the Newman-Penrose 
constants appear in a simple way in $\Phi_{-1}$ and $\Psi_{-2}$ (see Sec. 6).

\section{PEELING PROPERTIES}

There are three distinct types of infinities in an asymptotically flat space-time, corresponding to three possible choices of time coordinate. If $r \rightarrow \infty$ with the static time coordinate $t$ in the Schwarzschild metric held constant, the limit is called spacelike infinity. The limit $r \rightarrow \infty$ at constant retarded time $u$ is future null infinity, while the limit $r \rightarrow \infty$ at constant advanced time $v$ [see Eq. (2.3)] is past null infinity. Penrose $e^{9,10}$ has pioneered the study of the conformal structure of infinity. He transforms coordinates to bring $r=\infty$ in an asymptotically flat space-time to finite coordinate values and then removes the induced singularity in the metric by a conformal transformation. The original open, noncompact manifold $\bar{M}$ is converted to a manifold $M$ which contains future and past null infinity as regular null hypersurfaces $\left(g^{+}\right.$ and $\mathscr{F}$, respectively). Spacelike infinity is represented by a point $I_{0}$, which is generally a singular point of $M$. A spin-s zero-rest-mass field in the physical open, noncompact manifold $\bar{M}$ can be described by a totally symmetric spinor $\bar{\phi}_{A \cdots K}$, with $2 s$ indices. The corresponding conformally transformed spinor

$$
\phi_{A} \cdots_{K}=\Omega^{-(s+1)} \bar{\phi}_{A \cdots K}
$$

satisfies the spin-s zero-rest-mass field equation in $M$. If $\phi_{A} \cdots_{K}$ is continuous at $g^{-}$and $g^{+}$, the field is called asymptotically regular. Penrose ${ }^{9}$ shows that an asymptotically regular field has the following peeling behavior:

$$
\left(r^{s+p+1} \psi_{p}\right)
$$

has a limit at future null infinity, and

$$
\left(r^{s-p+1} \psi_{p}\right)
$$

has a limit of past null infinity, where $\psi_{p}$ is the spinweight- $p$ part of the field tensor. For an electromagnetic field $\psi_{p}=\Phi_{p}$ and for a gravitational field $\psi_{p}=\Psi_{p}$.

Penrose then prove asymptotic regularity of the gravitational field on the assumption that the geometry of the spacetime is sufficiently smooth at null infinity, specifically that the manifold $M$ is $C^{4}$-differentiable with a $C^{3}$ metric and that the conformal factor $\Omega$ is $C^{3}$ on $M$. (This step is close to a tautology, since the geometry is the gravitational field.) With the same regularity of the geometry an electromagnetic field is not required to be asymptotically regular-expressions (5.2) and (5.3) need only be bounded at future and past null infinity, respectively.

On the other hand, Couch and Torrence ${ }^{19}$ have shown that a very much weaker type of peeling behavior, in which $r^{s+1} \psi_{p}$ need not be bounded for $p \geq 0$ at future null infinity and for $p \leq 0$ at past null infinity, is consistent with asymptotic flatness.

How much regularity at future or past null infinity can be expected purely on the basis of a certain set of assumptions about the physical nature of the source? We begin an exploration of this question using our general retarded test field solutions.

The only restriction we impose in deriving the solutions are the absence of incoming radiation at past null infinity, the boundedness of the retarded multipole moment at all times to the past, and the validity of the test field approximation.

The first two restrictions correspond to the physical condition that the sources of the test fields be bounded within a compact region for all times to the past. If the source is contained within a radius $R>2 M$, a rough estimate of the maximum possible gravitational multipole moment is $M R^{l}$. Charge separation for electromagnetic (or possibly scalar) sources increases the limit to the order of $R^{l+1}$ (charges measured in Gaussian units), at which point the test field approximation breaks down.

The validity of the test field approximation requires that the nonlinear contributions of the test fields in the exact Einstein equations do not significantly modify the background Schwarzschild metric. The first $(l+s)$ derivatives of $A(u)$ must be bounded, so that the $\Psi_{p}(u, r)$ will be bounded. Also, the energy radiated over all times to the past must be small compared with the gravitational mass $M$. An explicit positive-definite ${ }^{20}$ expression for the energy radiated from a particular $2^{l}$-pole is

$\frac{1}{2 \pi}\left(2 ! \frac{(l-s) !}{(2 l) !}\right)^{2} \int_{-\infty}^{u}\left|A^{(l+1)}\left(u_{0}\right)^{2}\right| d u_{0} \ll M(u=-\infty)$.

We claim that no further constraints on $A(u)$ are physically necessary.

At future null infinity our general retarded solutions for scalar, electromagnetic, and gravitational fields are all asymptotically regular in the Penrose sense. Moreover, the coefficients of $r^{-(s+p+1)}$ in the $\psi_{p}$ are related to each other and (by definition) to $A(u)$ in the same way as in a flat background:

$$
\begin{aligned}
\lim _{r \rightarrow \infty} r^{s+p+1} \psi_{p}(u, r)=(-1) s-p & \left(2 s-p \frac{(l+p) !}{(l-p) !} \frac{(l-s) !}{(l+s) !}\right) 1 / 2 \\
& \times \frac{2^{l-s(l+s) !}}{(2 l) !} A^{(l-p)(u)}
\end{aligned}
$$

In the limit $r \rightarrow \infty$ Eqs. (2.16c) and (2.16d) and Eqs. (2.18e)-(2.18h) acting on the general retarded solution reduce to the flat-space equations. Note that Eq. (3.9) has been established by our proof of Eqs. $(3.46)$ and (3.47).

To find the peeling behavior at past null infinity, let

$$
u=v-2 r
$$

and take the limit $r \rightarrow \infty$ with $v$ constant. While $v$ is not the exact advanced time in the Schwarzschild background, it becomes exact in the limit. The backscatter part of the field $\psi_{\mathrm{II}}(u, r)$ is of order $(2 M / r)$ compared with $\psi_{I}(u, r)$ and does not contribute to $\Psi_{p}(u, r)$ in the limit. Also, we can neglect terms of $\operatorname{order}(2 M / r)$ in the coefficient of a given derivative of $A(u)$ in $\psi_{I}(u, r)$. Without assuming anything about relative magnitudes of the derivatives at past null infinity, we obtain

$$
\begin{aligned}
& r^{s-p+1} \psi_{p} \simeq 2^{(p-s) / 2} \lim _{r \rightarrow \infty} \sum_{k=0}^{l-p} \frac{(2 l-k) !}{(2 l) !} \frac{(l-p) !}{k !(l-p-k) !} 2^{k} \\
& \times r^{-l-p+k} A^{(k)}(v-2 \gamma) .
\end{aligned}
$$

The content of Eq. (5.7) is that the general retarded solu- 
tion in the Schwarzschild background approaches a retarded flat-space solution at past null infinity as well as at future null infinity.

Since the $A^{(k)}$ are bounded, $r^{s-p+1} \Psi_{p}$ approaches a limit of zero at past null infinity for $p>0$. However, $r^{s+1} \psi_{0}$ need only be bounded. The condition (5.4) on the radiated energy allows $r^{s-p+1} \psi_{p}$ to be unbounded at past null infinity for $p<0$. For instance,

$$
A(u) \sim \sin \left[b\left(u / u_{1}\right)^{1 / 3}\right]
$$

gives a finite energy radiated for all $l \geq s \geq 0$, but

$$
r^{2 s+1} \psi_{-s} \sim\left(r^{2 / 3}\right)(2 s-l) \rightarrow \infty
$$

at past null infinity for $0<s \leq l<2 s$.

A mathematical condition that the field be asymptotically regular at past null infinity is equivalent to a restriction on the time dependence of the multipole moment in the distant past which is much stronger than Eq. (5.4). For example, if $l=s$ the restriction is that

$$
\lim _{u \rightarrow-\infty} u^{k A}(k)(u)
$$

exist for $0 \leq k \leq 2 s$. The multipole moment must be asymptotically static in the infinite past-to any given accuracy it must be static for an infinite time. Such a restriction is not required by any physical regularity condition. We conclude that the geometrical regularity conditions assumed by Penrose are not physically necessary.

A physically more appropriate approach to peeling theorems is a direct argument from the general retarded solution to the field equations. Sachs ${ }^{21}$ and Goldberg and $\mathrm{Kerr}^{22}$ have proved such theorems for linearized gravitational fields and for electromagnetic fields in flat space. Our results support the proposition that backscatter cannot be strong enough in an asymptotically flat space-time to destroy the asymptotic regularity of the field at future null infinity.

\section{THE NEWMAN-PENROSE QUANTITIES}

The standard prescription for calculating the NPQ'S associated with a spin-s field $4,5,23$ is as follows. Consider the Newman-Penrose field scalar with spin weight $p=s$. Extract the lowest radiatable multipole $l=s$, by performing an angular integration. Denote the resulting function of $u$ and $r$ by $\psi_{s}(u, r)$. For example, in the gravitational case

$\psi_{s}(u, r)=2 \pi \int \sin \theta d \theta d \varphi \Psi_{2}(u, r, \theta, \varphi)_{2} Y_{m}^{2}(\theta, \varphi)$.

The $\psi_{s}(u, r)$ are really $2 s+1$ complex functions, corresponding to the $2 s+1$ possible values of the axial eigenvalue $m$. Now let

$$
P(u, r)=r^{2 s+1} \psi_{s}(u, r)
$$

and

$$
Q(u, r)=\frac{\partial}{\partial(1 / r)}\left[r^{2 s+1} \psi_{s}(u, r)\right]
$$

The lowest radiatable multipole moment is

$$
A_{s}(u)=\lim _{r \rightarrow \infty} P(u, r)
$$

and the NPQ is

$$
\mathrm{NPQ}=\lim _{r \rightarrow \infty} Q(u, r)
$$

Both limits are at future null infinity. There are $2(2 s+$ 1) NPQ's associated with the spin-s field, corresponding to the real and imaginary parts of the $(2 s+1)$ functions $\psi_{\mathrm{s}}$.

Newman and Penrose $e^{4,5}$ assume that

$\psi_{s}(u, r)=\frac{A_{s}(u)}{r^{2 s}+1}+\frac{\mathrm{NPQ}}{r^{2 s+2}}+\theta\left(r^{-(2 s+3)}\right)$,

so that both limits $(6.4)$ and $(6.5)$ exist, and then show that the gravitational NPQ's are conserved (independent of $u$ ) if the field satisfies the vacuum Einstein equations near null infinity. Exton, Newman, and Penrose ${ }^{23}$ prove that the electromagnetic and gravitational NPQ's are conserved by the vacuum Einstein-Maxwell equations in asymptotically flat space-times.

Subsequent papers ${ }^{24}$ have examined various mathematical properties of the NPQ's, but have not shed much light on their physical meaning. We try to fill this gap by asking the following equestions in the context of test fields in the Schwarzschild background: (i) Are the NPQ's measurable in any physically meaningful sense? (ii) Under what conditions does the limit (6.5), and therefor the NPQ, exist as a formal mathematical property of the test field? (iii) If the limit does exist, what is the physical interpretation of the value of the NPQ?

The answers, in brief, are: (i) The NPQ's are not measur able and, therefore, have no direct physical significance.

(ii) The NPQ's do not exist for all physically nonsingular retarded solutions to the field equations. (iii) When the NPQ does exist, its value is proportional to a certain average of the lowest radiatable multipole moment in the infinite past.

We begin with the general retarded solution for $\psi_{s}(u, r)$ as obtained in Sec. 3 :

$$
\begin{aligned}
& \psi_{s}(u, r)=A_{s}(u) r^{-(2 s+1)} \\
& \quad+r^{-(2 s+1)} \sum_{k=1}^{\infty} a_{k} g_{k}(u, r)\left(\frac{2 M}{r}\right)^{k} .
\end{aligned}
$$

Since the $g_{k}(u, r)$ are uniformly bounded if $A_{s}(u)$ is uniformly bounded to the past

$$
P(u, r)=A_{s}(u)+\vartheta(2 M / r) .
$$

If the general retarded solution (6.7) is substituted into Eq. (6.3) for $Q(u, v)$, the result is

$$
Q(u, r)=(2 s+1) M\left[\left(g_{1}-r \frac{\partial g_{1}}{\partial r}\right)+\theta(2 M / r)\right] .
$$

For general $s$ [see Eqs. (5.4a). (5.4b) and (4.17a), (4.17b)],

$$
g_{1}(u, r)=A_{s}(u)-\int_{-\infty}^{u} d u_{0} \frac{d A_{s}}{d u_{0}} h_{1}(y)
$$

with

$$
h_{1}(y)=\frac{1}{(2 s+1) y}\left(1-\frac{1}{(1+y)^{2 s+1}}\right)
$$

and

$$
y=\left(u-u_{0}\right) / 2 r .
$$


Note that $f_{l+s}\left(u_{0}\right)$ appears in Eq. $(3.38)$ for $g_{1}(u, r)$, vanishes identically when $l=p=s$ as a consequence of the peeling conditions at future null infinity. Equations (6.9)-(6.12) combine to give at $r \gg 2 M$.

$$
\begin{aligned}
& Q(u, r)=(2 s+1) M\left(A_{s}(u)\right. \\
& \left.\quad-\int_{-\infty}^{u} d u_{0} \frac{d A_{s}}{d u_{0}} \frac{1}{(1+y)^{2 s+2}}\right) \\
& \text { or }
\end{aligned}
$$

$$
\begin{aligned}
Q(u, r)=(2 s & +1)(2 s+2) M \\
& \times \int_{0}^{\infty} d y A_{s}(u-2 r y)(1+y)-(2 s+3)
\end{aligned}
$$

The integrals in Eqs. (6.10) and (6.13a) come from a superposition of incoming waves (the backscatter) generated by previous changes in the multipole moment.

Both $P(u, r)$ and $Q(u, r)$ are directly measurable by a network of observers covering a finite region of spacetime surrounding the source region at $r \gg 2 M$. The observers measure the field tensor as a function of position. They must project the tensor at each point on an appropriate null tetrad and then perform an angular integration to obtain $\psi_{s}(u, r)$ over the span of radius $r$ and over the span of retarded time $u$ covered by the observers. The choice of null tetrads is not unique in general; but the spherical symmetry allows a unique choice for the background Schwarzschild metric. To first order in the gravitational field perturbations only the spin-weight $p=0, \pm 1$ parts of the perturbed Weyl tensor are affected by the uncertainty in the tetrad induced by the first-order deviations from spherical symmetry. In all cases, then, $\psi_{s}(u, r)$ is unambiguous to first order in the test field. Given measurements of $\psi_{s}(u, r)$ for $l=s$ to some finite accuracy over a range of $r$ and $u$ the observers can extract the values of $P(u, r)$ and $Q(u, r)$ to a corresponding accuracy.

Equation (6.8) says that at values of $r \gg 2 M$ the observers will find that $P(u, r)$ is independent of $r$ along an outgoing radial null geodesic, and assures that its variation with $u$ can safely be interpreted as the variation of the multipole moment $A_{s}(u)$, as defined at future null infinity, with $u$. In this sense, the lowest radiatable multipole moment is measurable.

The Newman-Penrose constant is the limit (6.5) of $Q(u, r)$ at future null infinity. However, Eq. $(6.13)$ gives no assurance that the limit exists, let alone that the value of the limit can be extracted from measurements of $Q(u, r)$ over a finite region of space-time.

Consider as an example $A_{s}(u)=A_{1}$ for all $u<u_{1}$ and $A_{s}(u)=A_{2}$ for all $u>u_{1}$. Equation $(6.13)$ gives

$$
\begin{aligned}
& Q(u, r)=\left\{\begin{array}{l}
(2 s+1) M A_{1}, \quad u<u_{1} \\
(2 s+1) M\left\{A_{2}\right.
\end{array}\right. \\
& \left.-\left(A_{2}-A_{1}\right)\left[1+\left(u-u_{1}\right) / 2 r\right]^{-(2 s+2)}\right\}, \quad u>u_{1} .
\end{aligned}
$$

The NPQ at all $u$ is the intial static value of $Q$ given by Eq. (6.14a). However, at any fixed, finite value of $r, Q$ goes smoothly toward a new asymptotically static value appropriate to the new value of the multipole moment in the limit $u-u_{1} \gg 2 r$.

Measurements of finite accuracy will not detect any deviation from the new static value of $Q$ if the change in the multipole moment occurred at a time $u_{1}$ sufficiently far in the past, such that $u_{1} \ll u-2 r$. It is not physically reasonable to require that measurements be made infinitely far in the past $(u \rightarrow-\infty)$ or at infinitely large radii $(r \rightarrow \infty$ at finite $u)$, or that they be infinitely accurate. An apparently constant $Q$ need not be constant all the way out to future null infinity, so the value of $Q$ at future null infinity, the NPQ, is not measurable in a physically realistic sense.

As Eq. (6.13b) makes explicit, the value of $Q$ at given $u, r$ is proportional to a weighted time average of the multipole moment over the entire past history. The weighting function $(2 s+2)(1+y)^{-(2 s+3)}$ cuts off at $y \sim 1$ or $u_{0} \sim u-2 r$, so the average is effectively over a time $\Delta u=u-u_{0} \sim 2 r$ previous to the retarded time at which $Q$ is being evaluated. In the limit $r \rightarrow \infty$, the interval $\Delta u$ expands to cover the entire past history uniformly. The NPQ is a uniform average of $A_{s}\left(u_{0}\right)$ over the entire past, if the average exists. We shall see below that such an average may not exist. Since any finite range of $u_{0}$ makes a negligible contribution to the average over the entire past, the value of the NPQ, if it exists, cannot be extracted from measurements of the field at finite $u$ and $r$, which are only sensitive to $A_{s}\left(u_{0}\right)$ over a finite time to the past. In physical terms, the presence of an "average value" in the field is due to the local superposition of backscatter from the outgoing radiation of all previous retarded times.

We define a measurable Newman-Penrose quantity (MNPQ) to be the value of $Q$ in a region of space-time where $Q$, to the finite accuracy of the measurements, is a constant independent of $u$ and $r$. For an MNPQ to exist, the average value of $A_{s}\left(u_{0}\right)$ must have been constant over times $\Delta u \gg 2 r$ to the past. Either $A_{s}\left(u_{0}\right)$ itself was constant or substantial net deviations of $A_{s}\left(u_{0}\right)$ from the average value only lasted for a time $\delta u \ll 2 r$.

The above definition of an MNPQ differs from our previous $^{8}$ identification of the MNPQ as the coefficient of $r^{-(2 s+2)}$ in an asymptotic expansion of the field in powers of $\boldsymbol{r}^{-1}$. The old MNPQ was not defined very precisely mathematically, since an asymptotic expansion of the field in powers of $r^{-1}$ is not always possible. Once established, the old MNPQ does persist until a time $u-u_{1}$ $=2 r$ after the field becomes dynamic at $u=u_{1}$. The old MNPQ fails at the $\frac{2}{3}$-speed of-light cone $u \stackrel{1}{-} u_{1}=2 r$, because on this cone the maximum value of $y$ which appears in the integral $(6.13 \mathrm{a})$, with $A_{s}\left(u_{0}\right)$ static for $u_{0}<u_{1}$, is $y=1$. At $y=1$ an expansion of $(1+y)^{-(2 s+2)}$ in powers of $r^{-1}$ diverges.

The $\frac{1}{3}$-speed-of-light cone has no special meaning for the new MNPQ's. These are associated with the quantity $Q(u, r)$, which is always well defined and varies continuously when the multipole moment changes. For a given measurement accuracy $\epsilon$ the new MNPQ persists until $\left(u-u_{1}\right) / 2 r=O(\epsilon)$ after the multipole moment changes [see Eq. (6.14b), for example].

Goldberg ${ }^{25}$ has still another definition of MNPQ's which relates them to an artificially constructed "conserved flux." Goldberg's MNPQ's also change continuously when the multipole moment changes. Their values at finite $u$ and $r$ are no more closely related to the values of the NPQ's, if they exist, than our MNPQ's.

There is a limit on how rapidly $Q(u, r)$ can vary. Note that since $A_{s}(u)$ is bounded 


$$
\begin{aligned}
\frac{\partial Q}{\partial u} & =(2 s+1)(2 s+2) M \int_{0}^{\infty} d y A_{s}^{\prime}(u-2 r y)(1+y)-(2 s+3) \\
& =\theta\left(r^{-1} Q\right) .
\end{aligned}
$$

We now consider some examples of when the limit (6.5) defining the NPQ at future null infinity does and does not exist. First, a sufficient condition for the NPQ to exist is that the limit

$$
A_{s}(-\infty)=\lim _{u \rightarrow-\infty} A_{s}(u)
$$

exist. Then the average value of $A_{s}(u)$ in the limit $r \rightarrow \infty$ in Eq. $(6.13 \mathrm{~b})$ is just $A_{s}(-\infty)$, and

$$
\mathrm{NPQ}=(2 s+1) M A_{s}(-\infty) \text {. }
$$

A multipole moment which has the limit $(6.16)$ is, by definition, asymptotically static in the infinite past.

A retarded solution to the test field equations which satisfies the strong Penrose peeling condition at past null infinity is asymptotically static in the infinite past and, therefore, possesses NPQ's. However, we have seen in Sec. 5 that there are no physical restrictions which require the field to satisfy the Penrose peeling condition at past null infinity or to be asymptotically static in the infinite past.

As an example of a solution which is not asymptotically static in the infinite past, but still possesses NPQ's, consider

$$
A_{s}(u)=\sin b u
$$

for a scalar field $(s=0)$. The integrals in Eqs. (6.13a) or $(6.13 \mathrm{~b})$ for $Q(u, r)$ involve sine and cosine integrals. Let and

$$
\operatorname{Si}(x)=\frac{1}{2} \pi-f(x) \cos (x)-g(x) \sin (x)
$$

$$
\mathbf{C i}(x)=\gamma+\ln (x)+f(x) \sin (x)-g(x) \cos (x) .
$$

The functions $f(x)$ and $g(x)$ have asymptotic expansions

$$
f(x) \simeq \frac{1}{x}\left(1-\frac{2 !}{x^{2}}+\frac{4 !}{x^{4}}-\cdots\right)
$$

and

$$
g(x) \simeq \frac{1}{x^{2}}\left(1-\frac{3 !}{x^{2}}+\frac{5 !}{x^{4}}-\cdots\right)
$$

when $x \gg 1$. The result for $Q$ is

$$
\begin{aligned}
& Q(u, r)=\left[1-(2 r b)^{2} g(2 r b)\right] \sin (u) \\
& -(2 r b)[1-(2 r b) f(2 r b)] \cos (u) .
\end{aligned}
$$

In the limit $r \rightarrow \infty$

$$
Q(u, r) \simeq-\frac{\cos (u)}{b r}+\theta\left(\frac{1}{(b r)^{2}}\right) \rightarrow 0,
$$

so the NPQ exists and equals zero. In other words, the average of the multipole moment can approach a limit as $u_{0} \rightarrow \infty$, even though the multipole moment itself does not.

If the multipole moment varies on time scales which are the order of $u-u_{0}$ in the limit $u_{0} \rightarrow-\infty$, the NPQ does not exist. For instance, if

$$
A_{s}(u)=\sin \left(c \sinh ^{-1} b u\right)
$$

and $c \ll 1, A_{s}\left(u_{0}\right)$ is approximately constant over most of the range $u>u_{0}>u-2 r$ when $2 r b \gg 1$. Thus

$Q(u, r) \simeq(2 s+1) M\left\{\sin \left[b \sinh ^{-1}(-2 r b)\right]+\theta(c)\right\}$

and oscillates indefinitely in the limit $2 r b \rightarrow \infty$.

We conclude that the NPQ exists if and only if the average $A_{s}\left(u_{0}\right)$, over a time $\Delta u=u-u_{0}$, approaches a limit as $u_{0} \rightarrow-\infty$. Either $A_{s}$ is asymptotically static in the infinite past, or the time variation of $A_{s}$ is entire$l y$ on time scales infinitesimally short compared with $u-u_{0}$ in the limit $u_{0} \rightarrow-\infty$. Both of these conditions are rather special, and the NPQ's will not exist for the generic retarded test field solution.

The conservation of the NPQ's, when they exist, has no predictive powers; it is a reflection of the fact that an average of $A_{s}\left(u_{0}\right)$ over an infinite time is not affected by time variations over any finite time span. Therefore, the existence of the NPQ at any finite retarded time automatically implies the existence of the NPQ with the same value at any other finite retarded time. The value of the field at finite $u$ and $r$ depends, to any finite accuracy, only on the multipole moment over a finite range of retarded time to the past and is, thus, in principle completely independent of the value of the NPQ.

We are left with NPQ's which, when they exist, have only a formal mathematical significance. For a spin-s zerorest-mass test field in any static, spherically symmetric, asymptotically flat background (in any metric theory of gravity), this mathematical significance has a simple origin. As long as the metric coefficients for the static background are analytic in $1 / r$ in some neighborhood of $1 / r=0$, it is possible to expand the general retarded solution for the spin-weight $p=s, l=s$ part of the field as

$\psi_{s}=A_{s}(u) r^{-(2 s+1)}+r^{-(2 s+1)} \sum_{n=1}^{\infty} a_{k}\left(\frac{2 M}{r}\right)^{k} g_{k}(u, r)$.

Here $M$ is just a parameter indicating the scale of radius on which the deviations from flat space become large; it need not have an interpretation as a gravitational mass. The $a_{k}$ are coefficients chosen so that the static solution for $\psi_{s}$ has $g_{k}=A_{s}=$ const. The relativistic equations may couple $g_{k}(u, r)$ to $g_{k-2}, g_{k-3}$, etc. as well as the $g_{k-1}$. However, $g_{1}(u, r)$ can only couple to $f_{2 s+1}(u)$, as before, since the $f_{n}(u)$ with $n<2 s+1$ are identically zero. The function $H_{1,1}(y)$ in this context, also, is the homogeneous similarity solution to the flatspace spin-s field equations. In general, then,

$Q(u, r)=2(2 s+2) M a_{1}$

$\times\left[\int_{0}^{\infty} d y A_{s}\left(u_{0}=u-2 r y\right)(1+y)^{-(2 s+3)}+o\left(\frac{2 M}{r}\right)\right]$.

The existence and value of the NPQ is related to an average over the lowest radiatable multipole moment in the infinite past in essentially the same way as before. The only possible difference is the value of the coefficient $a_{1}$ in the relativistic static solution. Thus, the conservation of the NPQ's (when they exist) depends only on asymptotic flatness; it is independent of any special properties of the curvature correction to the field equations. 
An apparent special property of the Einstein-Maxwell equations for test fields in the Schwarzschild background is the appearance of the NPQ's in the spin weight $p=-s$ part of the lowest radiatable multipole. In Eq. (3.38), for example, $f_{l+s}$ is not identically zero when $l=s=-p$; but it has a vanishing coefficient and does not contribute to $g_{k}(u, r)$. However, even this is a result only of asymptotic flatness, plus consistency of the equations for the different spin-weight parts of the field. The leading backscatter at future null infinity can always be interpreted as an incoming wave in flat space, so the coefficients of the incoming wave in the different spinweight parts of the field must be related by the flatspace equations. If the backscatter compensates for the changes in multipole moment in $g_{1}(u, r)$ of the spin weight $p=s$ part of the field, as it does when $l=s$, it must do so in $g_{1}(u, r)$ of the other spin-weight parts of the field, as well.

The generalization of our results to asymptotically flat solutions of the full nonlinear Einstein and EinsteinMaxwell field equations is not quite as straightforward. For instance, the NPQ's of the gravitational field have a different form when a dynamic electromagnetic field is present. ${ }^{23}$ It does seem safe to conclude that if the lowest radiatable multipole moments of the electromagnetic and gravitational fields do not have the asymptotic behavior in the infinite past necessary for the existence of the test field NPQ's, the NPQ's of the respective fields will not exist in the full nonlinear theory either. The conservation of the NPQ's, when they exist, is probably as trivial a consequence of asymptotic flatness as it is for test fields.

It may be possible to obtain general retarded solutions to the exact field equations at large $r$ similar to our test-field equations and check the validity of these conjectures directly. Care must be taken not to assume more regularity at future or past null infinity than is physically justified.

Our approach to the physical interpretation of the NPQ's has concentrated on their existence and measurability. Glass and Goldberg ${ }^{24}$ have interpreted the conservation of the NPQ's in terms of invariant transformations and an artificially constructed differential conservation law. They assume that the NPQ's exist and then show that their conservation is related to a superposition principle for ingoing and outgoing waves valid asymptotically in the lowest radiatable multipole in asymptotically flat space times. We have not found any physical content to the "conserved flux" they define.

\section{SUMMARY AND CONCLUSION}

Using the general retarded solution of our master equation for the radiative parts of test fields in the Schwarzschild background, we have examined the nature of the fields' Newman-Penrose quantities and peeling properties.

The explicit retarded test-field solution shows that the NPQ's are a certain average of the lowest radiatable multipole moment over the infinite past and do not exist unless the average exists. Even when they do exist, the NPQ's are not measurable and, therefore, have no direct physical significance.

Of course, the asymptotically flat boundary condition is an abstraction which ignores the existence of other matter in the universe. In practice, for a star in the galaxy, one can ignore the other stars out to a radius of about one light year at most. At such a radius the multipole moments are very well established, since if $M=1 M_{\odot}, 2 M / r$ is the order of $10^{-12}$. However, any net change in the lowest radiatable multipole moment on a year's time scale or longer makes it impossible to talk about a conserved NPQ.

The peeling properties of test fields in the Schwarzschild background are identical to the peeling properties in a flat background. The mathematical regularity assumptions of the Penrose peeling theorem are justified at future null infinity, but not at past null infinity.

The general retarded solution can also be used to study the detailed development and decay of the backscatter and wavetails for all radiatable multipoles. The wavetail is outgoing radiation at future null infinity at retarded times after the source has become static. This material, however, will be presented in a subsequent paper.

\section{ACKNOWLEDGMENT}

We thank Kip Thorne and Richard Price for introducing us to this problem and for many helpful discussions. This work was begun while one of us (J.M.B.) was visiting Caltech as a Senior Research Associate in the spring of 1971 .

*Supported in part by the National Science Foundation (GP-15267) at the University of Washington and (GP-27304, GP-28027) at the California Institute of Technology.

$\uparrow$ Present address: Yale University, New Haven, Connecticut. $\ddagger$ Fannie and John Hertz Foundation Fellow.

'W. Kundt and E. T. Newman, J. Math. Phys. 9, 2193 (1968)

${ }^{2}$ R. G. McLenaghan, Proc. Camb. Philos. Soc. 65, 139 (1969).

${ }^{3}$ W. B. Bonner and M. A. Rotenberg, Proc. R. Soc. A 289, 247 (1965).

${ }^{4}$ E. T. Newman and R. Penrose, Phys. Rev. Lett. 15, 231 (1965).

${ }^{5}$ E. T. Newman and R. Penrose, Proc. R. Soc. A 305, 175 (1968).

${ }^{6}$ R. H. Price, Phys. Rev. D 5, 2419 (1972).

${ }^{7}$ R. H. Price, Phys. Rev. D 5, 2439 (1972).

${ }^{8}$ W. H. Press and J. M. Bardeen, Phys. Rev. Lett. 27, 1303 (1971).

${ }^{9}$ R. Penrose, Proc. R. Soc. A 284, 159 (1965)

${ }^{10} \mathrm{R}$. Penrose, in Relativity, groups, and topology, edited by C. DeWitt and B. DeWitt (Gordon and Breach, New York, 1964).

${ }^{11}$ E. T. Newman and R. Penrose, J. Math. Phys. 3, 566 (1962).

${ }^{12}$ E. T. Newman and R. Penrose, J. Math. Phys. 7, 863 (1966).

${ }^{13}$ A. I. Janis and E. T. Newman, J. Math. Phys. 6, 902 (1965).

${ }^{14}$ T. Regge and J. A. Wheeler, Phys. Rev. 108, 1063 (1957).

${ }^{15}$ F. J. Zerilli, Phys. Rev. D 2, 2141 (1970).

${ }^{16} \mathrm{~K}$. S. Thorne, in Magic without magic: John Archibald

Wheeler, edited by J. Klauder (Freeman, San Francisco, 1972).

${ }^{17}$ W. E. Couch and R. J. Torrence, J. Math. Phys. 9, 484 (1968).

${ }^{18}$ W. E. Couch and W. H. Halliday, J. Math. Phys, 12, 2170 (1971).

${ }^{19}$ W. E. Couch and R. J. Torrence, J. Math. Phys. 13, 69 (1972).

${ }^{20} \mathrm{H}$. Bondi, M. van der Burg, and A. Metzner, Proc. R. Soc. A 269, 21 (1962)

${ }^{21}$ R. K. Sachs, Proc. R. Soc. A 264, 309 (1961); Proc. R. Soc. A 270, 103 (1962); Phys. Rev. 128, 2851 (1962)

${ }^{22}$ J. N. Goldberg and R. P. Kerr, J. Math. Phys. 5, 172 (1964).

${ }^{23}$ A. R. Exton, E. T. Newman, and R. Penrose, J. Math. Phys. 10, 1566 (1969).

${ }^{24}$ For references see E. N. Glass and J. N. Goldberg, J. Math. Phys 11, 3400 (1970).

${ }^{25}$ J. N. Goldberg, Phys. Rev. Lett. 28, 1400 (1972). 